\title{
Derived telemetry-based electrocardiograms in the intensive care unit: insights from a COVID-19 epicenter
}

\author{
Ellie Coromilas ${ }^{1}$, Daniel Pugliese ${ }^{1}$, Stephanie Kochav ${ }^{1}$, Hirad Yarmohammadi ${ }^{1}$, Elaine \\ $\mathrm{Wan}^{2}$, and Jose Dizon ${ }^{1}$ \\ ${ }^{1}$ Columbia University \\ ${ }^{2}$ Columbia University Medical Center
}

June 10, 2020

\begin{abstract}
Introduction: Severely ill inpatients with SARS-CoV-2 infection, Coronavirus Disease 2019 (COVID-19) require close electrocardiographic (ECG) monitoring due to frequent cardiac involvement of the disease and cardiovascular side effects of therapies. This study aimed to compare ECG parameters measured from conventional 12-lead ECGs to those from a telemetry-generated 7-lead or single lead ECG to determine if the latter may be an alternative for screening and monitoring patients, particularly during a pandemic. Methods and Results: We identified 33 patients with respiratory failure due to COVID-19 undergoing telemetry monitoring in the intensive care unit. Each received a 12-lead ECG utilizing standard lead placement. A concurrent 7-lead ECG and single lead (lead II) tracing were obtained using the central telemetry system. Each ECG was interpreted and intervals manually measured by 2 cardiologists with disagreements adjudicated by a third. Compared to the 12-lead ECG measurement, the 7-lead ECG underestimated the corrected QT by on average $13.45 \pm 32.05 \mathrm{msec}$, and the single lead ECG underestimated corrected QT by $19.62 \pm 33.19 \mathrm{msec}$ (Bazett, p < 0.05). Bland Altman analysis also demonstrated evidence of a positive bias, suggesting that the telemetry-derived tracings underestimated the QT interval. The presence of $\mathrm{T}$ wave abnormalities and ST segment changes were overestimated by the telemetry-derived tracings as compared to standard ECGs. Conclusion: Though telemetry-derived ECGs may be useful in screening patients for significant ECG abnormalities, they likely do not represent a reliable replacement of the standard 12-lead ECG in the routine diagnosis and management of critically ill patients.
\end{abstract}

\section{Introduction}

Infection due to Severe Acute Respiratory Syndrome Coronavirus-2 (SARS-CoV-2) has infected over 5 million people worldwide, resulting in over 300,000 deaths. ${ }^{1}$ The illness caused by SARS-CoV-2, Coronavirus Disease 2019 (COVID-19) predominantly affects the respiratory system, however increasing data suggest that cardiovascular involvement is common and associated with poor outcomes. ${ }^{2}$ Additionally, commonly used therapies to treat COVID-19 may have unintended cardiovascular side effects such as cardiac arrhythmias. Early in the pandemic, the treatment regimen of hydroxychloroquine and azithromycin had been widely-adopted based on early studies suggesting improved clinical outcomes and viral clearance. ${ }^{3-5}$ Both of these medications are known to prolong the QT interval, leading to concerns regarding an elevated risk of arrhythmia, especially in patients with baseline electrocardiographic (ECG) abnormalities. ${ }^{6}$

Given the cardiac implications of both the infection, associated therapies, and underlying comorbid cardiovascular conditions, many patients in the ICU require assessment and continued monitoring of ECG parameters such as QT interval, ST segment changes, and QRS morphology, typically using a standard 12-lead ECG. However, concerns regarding healthcare provider exposure to COVID-19 and limited availability of personal protective equipment have led providers to seek alternative means to safely monitor patients while limiting 
the amount of time and number of caregivers entering a room. Current telemetry systems have the ability to produce a multi-lead ECG that is mathematically derived utilizing fewer electrodes than the conventional 12-lead ECG. It is unclear if derived technology may offer a safe and accurate means to assess and monitor patients for ECG abnormalities or interval ECG changes while minimizing provider exposure to COVID-19.

This study aims to compare ECG parameters measured from conventional 12-lead ECG to those derived from a telemetry-generated 7-lead or single lead tracing to determine if the latter may be a reasonable alternative for screening and monitoring patients, particularly during a pandemic.

\section{Methods}

We identified individuals with respiratory failure due to COVID-19 infection who were undergoing telemetry monitoring in the ICU and were planned to undergo evaluation with a 12-lead ECG. Subjects were included in the study if they had 12-lead and 7-lead ECGs obtained within a single 24-hour period and confirmed positive SARS-CoV-2 RT-PCR nasal swab. The study was approved by the Institutional Review Board at Columbia University Irving Medical Center.

Each subject received a 12-lead ECG at standard $(25 \mathrm{~mm} / \mathrm{sec})$ speed and normal voltage, utilizing standard lead placement (GE MAC 5500, GE Medical Systems, Milwaukee, WI). A 10 second 7-lead ECG and single lead (lead II) tracing were also obtained from the central telemetry system (Philips Intellivue, Koninklijke Philips Electronics, Netherlands). This system uses the EASI System that derives up to 7 leads from 5 electrodes positioned on the torso, including all 3 limb leads, 3 augmented leads, and 1 precordial lead (typically lead V1). ${ }^{7}$

Each 12-lead standard ECG, 7-lead telemetry-derived ECG, and Lead II tracing was interpreted and manually-measured by 2 independent cardiologists. Disagreements in reads were adjudicated by an independent third reader. The qualitative assessment or quantitative measurement that was agreed upon by at least 2 of the cardiologists was recorded as the final interpretation. Diagnoses evaluated included underlying rhythm, QRS axis, cardiac intervals (RR interval, PR interval, QRS interval, and QT interval), conduction abnormalities, the presence of $\mathrm{T}$ wave or ST segment abnormalities, and the presence of low voltage complexes. Automated measurements by the ECG computer system for rate and common intervals were also recorded. The QT interval was measured in 3 consecutive cycles on leads II, III, and the precordial leads (V5, V6 when available) with the maximal interval taken. The end of the $\mathrm{T}$ wave was defined as the tangent from the steepest last limb of the $\mathrm{T}$ wave to its intersection with the baseline. For irregular rhythms, a weighted average RR interval was calculated. ${ }^{8}$ QT interval correction was calculated using commonly-used formulae: Bazett, Fridericia, Framingham, and Hodges. QT dispersion was calculated as the difference between the longest and shortest measured QT intervals on available leads. Significant ST segment changes were considered to be more than $1 \mathrm{~mm}$ of elevation or depression. Low voltage was considered any ECG where the QRS was less than $5 \mathrm{~mm}$ in all of the limb leads or $10 \mathrm{~mm}$ in the precordial leads. Clinical information was obtained from the electronic medical record including demographics, comorbid conditions, laboratory parameters, and pertinent therapies.

Statistical analysis was performed using SAS version 9.4 (SAS Institute Inc., Cary, NC, USA). For continuous variables, means and standard deviations were calculated. For categorical variables, frequency and percentage were calculated. The differences in QT duration between the 12-lead ECG and both the derived 7-lead ECG and single-lead telemetry were calculated using students t-test. Pearson's correlation coefficients were calculated between ECG measurement methods and scatter plots were generated. Bland-Altman analyses were performed to evaluate agreement between measurement methods. ${ }^{9}$

\section{Results}

Thirty-three subjects were identified and included in the analysis. Patient demographics, medical history, laboratory data and clinical features are provided in Table 1. Overall, the population was $42.4 \%$ female with a mean age of $60.1 \pm 14.1$ years. Obesity and other cardiovascular comorbidities were common (e.g. $47.1 \%$ were obese with BMI $>30,63.6 \%$ had hypertension, $48.5 \%$ diabetes mellitus, and $12.1 \%$ had coronary 
artery disease). The study population was critically ill with all subjects receiving mechanical ventilation, approximately $66 \%$ receiving renal replacement therapy, and nearly $75 \%$ requiring vasopressors. The majority of patients were treated with hydroxychloroquine $(78.8 \%)$ and azithromycin (81.8\%).

The median amount of time between obtaining the 12-lead ECG and telemetry-derived ECG was 53 minutes (range 3 minutes to 13 hours and 16 minutes). ECG parameters measured from each ECG method are reported inTable 2 . The mean uncorrected QT interval in milliseconds (msec) for the group as a whole was $398.6 \pm 64.8,401.2 \pm 66.1,388.2 \pm 64.6$ and $382.1 \pm 60.7$ by computer measurement on 12-lead, manual measurement on 12-lead, 7-lead derived ECG, and telemetry lead II, respectively. Similarly, the mean corrected QT interval by Bazett formula in msec was 474.2 $\pm 39.4,467.5 \pm 50.9,454.1 \pm 49.4$ and $447.9 \pm 47.3$ by computer measurement on 12-lead, manual measurement on 12-lead, 7-lead derived ECG, and telemetry lead II, respectively. These measurements for the entire group were statistically similar.Table 3 shows the mean individual differences in QT measurements between the manual 12-lead ECG and both the 7-lead derived ECG and single-lead telemetry. The mean difference in measured QT was significantly shorter on both the 7-lead and single lead derived ECGs compared with the 12-lead ECG (p value for all comparisons $<0.05$ ). The mean QT difference was significantly larger between the 12-lead ECG and single lead telemetry than between the 12-lead ECG and 7-lead derived ECG.

Table 4 shows the morphologic features of ECGs. Thirty-one (93.94\%) patients were in sinus rhythm while 2 patients were in atrial fibrillation. Twenty-nine (87.88\%) subjects were identified as having a normal axis on the 12-lead ECG, compared to $26(78.79 \%)$ on the 7-lead ECG. Five (15.15\%) subjects had conduction delay on the 12-lead ECG; 2 had right bundle branch block (RBBB), 1 subject had left bundle branch block (LBBB), and 2 patients had nonspecific intraventricular conduction delay (IVCD). Using both the 7-lead ECG and telemetry lead II, $4(12.12 \%)$ subjects were identified as having conduction delay. Eleven (33.33\%) subjects had $\mathrm{T}$ wave inversion on the 12-lead ECG, compared with $14(42.42 \%)$ on the 7-lead ECG and 9 $(27.27 \%)$ on single lead telemetry. One (3.03\%) subject had ST depression and none had ST elevation on the 12-lead ECG, compared to $4(12.12 \%)$ subjects with ST depression and $5(15.15 \%)$ with ST elevation on the 7-lead ECG, and 1 (3.03\%) subject with ST depression and $2(6.06 \%)$ with ST elevation on telemetry lead II. Seven (21.21\%) subjects had low voltage QRS on the 12-lead ECG, compared to $2(6.06 \%)$ on the 7-lead ECG and $1(3.03 \%)$ on telemetry lead II. Figure 1 provides a representative example of a 12-lead and 7-lead ECG of the same patient, with less evident conduction delay and more pronounced ST segment changes on the 7-lead ECG.

Manually-measured QTc from standard 12-lead ECG was strongly linearly correlated with the manuallymeasured QTc from the 7-lead ECG and telemetry lead II, as well as the automatic computer-generated 12lead QTc measurement $(\mathrm{p}<0.0001)$ (Figure 2 ). Bland-Altman plots show a reasonable amount of agreement among ECG methods for QT measurement (Figure 3 ). There is evidence for a positive bias, consistent with an underestimation of the QT interval when measured on the telemetry-derived ECGs as compared with the standard 12-lead ECG. These differences in measurement were frequently between 25 and 50 msec and were up to $110 \mathrm{msec}$ when using the single lead tracing. Additional correlation and Bland-Altman plots for the remaining ECG parameters are provided in the Supplemental Material. A consistent positive bias was identified when comparing the corrected QT intervals between 12-lead and telemetry-derived ECGs.

\section{Discussion}

We evaluated the use of a telemetry-derived ECG in the evaluation of individuals admitted to the ICU with COVID-19 infection. The population studied had a high burden of comorbid cardiac disease, and the majority were treated with hydroxychloroquine and azithromycin, requiring close cardiac monitoring of QT interval for concern of risk of torsade de pointes. Our results suggest that telemetry derived ECGs cannot replace a standard 12-lead ECG, both for interval measurements and morphologic assessment.

The mean QT or QTc intervals for the entire group as measured on a 12-lead ECG, 7-lead derived ECG, or single-lead tracing were similar, which is not unexpected given small differences in measurements in a small 
population. Though there was a strong linear correlation between the QT interval as measured on the different tracings, the correlation was less robust when using the single-lead tracing. The method of using Pearson correlation only measures the magnitude of a relation between variables, and not the agreement between two methods. Accordingly, one would expect a high magnitude of correlation when making measurements of the same QT intervals. The Bland-Altman method of agreement plots is an established method for quantifying agreement between two quantitative measurements. When we evaluated the agreement between measurements of the QT intervals on a standard 12-lead ECG and telemetry-derived tracings, a positive bias was found, suggesting that the telemetry tracings tend to underestimate the "true" QT interval, as defined by the manually-measured QT on a standard 12-lead ECG. This finding was also demonstrated when we averaged the individual differences between the corrected QT intervals on derived leads and the manual 12 lead measurement, with an underestimation of QT interval by both derived measurements and a significantly lower value by the single lead method. We observed differences in measurements often between 25 and 50 msec, and up to 110 msec when using the single lead tracing.

There was significantly decreased QT dispersion when using the telemetry derived 7-lead ECG, which is likely explained by fewer leads available for measurement. This finding may account for the tendency to underestimate the QT interval when using the telemetry-derived ECGs, and is consistent with other studies that have attempted to identify alternative means to monitor patients during the COVID pandemic. When comparing the use of a handheld ECG device to a standard 12-lead ECG to assess QT intervals, Cheung, et al. concluded that while QT was similar when the device was used across multiple positions, interpretation of a single lead consistently led to underestimation of the QT interval. ${ }^{10}$

Expert guidance has suggested that the risk of treatment with hydroxychloroquine and azithromycin may outweigh the benefit in patients with a QT interval longer than 500msec. ${ }^{11}$ Given a mean baseline QTc interval of $467 \mathrm{msec}$, variance in the measured QT interval of the magnitude suggested in our cohort may lead to inappropriate treatment with QT-prolonging medications.

Other studies have suggested that mobile cardiac outpatient telemetry may be used for QT and arrhythmia monitoring during the COVID pandemic. ${ }^{12,13}$ However, these studies did not compare data to the standard 12-lead ECG, and our findings are consistent with a prior study comparing QTc measurements using single lead portable ECG devices and 12-lead ECG. ${ }^{14}$ A multi-lead tracing may allow for increased diagnostic accuracy. Prior comparisons of telemetry-derived ECGs and 12-lead ECGs also found moderate correlation between QTc measurements using these modalities, though had conflicting conclusions as to whether or not the telemetry-derived ECGs offered an acceptable alternative to the standard ECG. ${ }^{15,}{ }^{16}$ None of these comparisons assessed the ability of these alternative ECG monitors to assess other ECG parameters such as morphology or ST segment changes.

In our analysis, there was acceptable agreement in diagnoses among the different ECG modalities, though with some notable limitations. Although in general there was agreement in the QRS axis, $3(9.09 \%)$ subjects with normal axis were incorrectly identified as having a rightward or northwest axis using the 7-lead derived ECG. This may be attributable to inconsistent telemetry electrode placement leading to systematic error in the mathematical derivation.

There was a suggestion of decreased sensitivity for identifying low voltage QRS complexes on the 7-lead and single lead ECGs, which may be due to the lack of precordial leads for interpretation. Conversely, a larger number of subjects were identified as having T wave inversion or ST segment changes on the 7-lead or single-lead ECG than on the standard 12-lead ECG. This finding is not consistent with prior comparisons of the ESAI configuration with a standard 12-lead ECG, which found comparable incidence and location of ST segment changes in the setting of acute MI. ${ }^{17}$

Our findings suggest that single lead tracings are quite limited and may not be an adequate replacement for traditional ECG monitoring given the inability to define axis or morphology, a high likelihood of underestimating the QT interval, and overestimation of ST segment changes. Although utilizing a 7-lead tracing may allow additional accuracy, there were still significant limitations in interval measurement and the identifica- 
tion of morphologic changes. We did not evaluate serial changes on telemetry derived ECGs, however our results call into question whether changes in QT intervals can be reliably monitored with telemetry-derived ECGs. Based on our results, it appears prudent that a baseline 12-lead ECG should be performed, as well as a new 12-lead after any dose change of QT prolonging drug or significant clinical event.

Our study has several strengths in its methodology. We rigorously evaluated the ECG measurements by having two independent readers with disagreements adjudicated by a third reader. This study was performed in a real-world setting; we included consecutive admissions to two cardiac care units and did not exclude any 12-lead ECGs or telemetry ECGs if they were readable. Our statistical approach used multiple validated methods for assessing the agreement between our measurements, including use of the Bland-Altman agreement plots to quantify and visualize differences between pairs of measurements. While this study was motivated by a novel use of the ICU telemetry system during the time of a pandemic, it has broader implications for the use of the telemetry system as a replacement for routine electrocardiograms in a wide array of cardiovascular and acute care conditions.

There are several limitations to this study. Though attempts were made to collect ECG and telemetry strips at close intervals, not all of the tracings were performed simultaneously, which may lead to minor differences in morphology and intervals among ECG and telemetry strips. Additionally, variation in telemetry electrode placement may lead to error in the mathematical formulas used to derive the telemetry ECG, and correct electrode placement could not be verified although ICU staff are well trained in correct lead placement. Finally, the current study only assesses for differences between ECG parameters at one point in time. We did not assess serial ECGs in the same patient, thus our results cannot be extrapolated to the utilization of telemetry-derived ECGs to monitor for changes in intervals or morphology.

\section{Conclusion}

The present study suggests that the use of telemetry-derived ECGs may lead to underestimation of the true QT interval and presence of low voltage QRS, while overestimating the presence of $\mathrm{T}$ wave and ST segment abnormalities. While telemetry-derived ECGs may be useful in screening patients for significant ECG abnormalities, they likely do not represent a reliable replacement for the standard 12-lead ECG in the routine diagnosis and management of critically ill patients.

\section{References:}

1. Coronavirus Disease 2019 (COVID-19) Situation Report 124. 2020; https://www.who.int/docs/defaultsource/coronaviruse/situation-reports/20200523-covid-19-sitrep-124.pdf?sfvrsn=9626d639_2, Accessed May $24,2020$.

2. Driggin E, Madhavan MV, Bikdeli B, Chuich T, Laracy J, Bondi-Zoccai G, Brown TS, Nigoghossian C, Zidar DA, Haythe J, Brodie D, Beckman JA, Kirtane AJ, Stone GW, Krumholz HM and Parikh SA. Cardiovascular Considerations for Patients, Health Care Workers, and Health Systems During the Coronavirus Disease 2019 (COVID-19) Pandemic. J Am Coll Cardiol . 2020.

3. Colson P, Rolain JM, Lagier JC, Brouqui P and Raoult D. Chloroquine and hydroxychloroquine as available weapons to fight COVID-19. Int J Antimicrob Agents . 2020:105932.

4. Gao J, Tian Z and Yang X. Breakthrough: Chloroquine phosphate has shown apparent efficacy in treatment of COVID-19 associated pneumonia in clinical studies. Biosci Trends . 2020;14:72-73.

5. Gautret P, Lagier JC, Parola P, Hoang VT, Meddeb L, Mailhe M, Doudier B, Courjon J, Giordanengo V, Vieira VE, Dupont HT, Honore S, Colson P, Chabriere E, La Scola B, Rolain JM, Brouqui P and Raoult D. Hydroxychloroquine and azithromycin as a treatment of COVID-19: results of an open-label non-randomized clinical trial. Int J Antimicrob Agents . 2020:105949.

6. Szekely Y LY, Shrkihe BA, Bruck H, Oster HS, Viskin S. Chloroquine-induced torsade de pointes in a COVID-19 patient.Heart Rhythm . 2020. 
7. Dower GE, Yakush A, Nazzal SB, Jutzy RV and Ruiz CE. Deriving the 12-lead electrocardiogram from four (EASI) electrodes. J Electrocardiol . 1988;21 Suppl:S182-7.

8. Ehlert FA, Goldberger JJ, Rosenthal JE and Kadish AH. Relation between QT and RR intervals during exercise testing in atrial fibrillation. Am J Cardiol . 1992;70:332-8.

9. Bland JM and Altman DG. Statistical methods for assessing agreement between two methods of clinical measurement. Lancet . 1986;1:307-10.

10. Cheung CC DB, Gibbs K, Laksma ZW, Krahn AD. Multi-lead QT Screening is Necessary for QT measurement: Implications for Management of Patients in the COVID-19 Era. JACC Clin Electrophysiol . 2020 .

11. HRS COVID-19 Task Force Update: April 7, 2020. 2020.

12. Chang D, Saleh M, Gabriels J, Ismail H, Goldner B, Willner J, Beldner S, Mitra R, John R and Epstein LM. Inpatient Use of Ambulatory Telemetry Monitors for COVID-19 Patients Treated with Hydroxychloroquine and/or Azithromycin. J Am Coll Cardiol . 2020.

13. Gabriels J, Saleh M, Chang D and Epstein LM. Inpatient use of mobile continuous telemetry for COVID19 patients treated with hydroxychloroquine and azithromycin. HeartRhythm Case Rep . 2020.

14. Bekker CL, Noordergraaf F, Teerenstra S, Pop G and van den Bemt BJF. Diagnostic accuracy of a singlelead portable ECG device for measuring QTc prolongation. Ann Noninvasive Electrocardiol . 2020;25:e12683.

15. Janssen GH, Rijkenberg S and van der Voort PH. Validation of continuous QTc measurement in critically ill patients. J Electrocardiol . 2016;49:81-6.

16. Chenoweth JA, Hougham AM, Colby DK, Ford JB, Sandhu J, Albertson TE and Sutter ME. Monitoring the corrected QT in the acute care setting: A comparison of the 12lead ECG and bedside monitor. Am $J$ Emerg Med . 2018;36:777-779.

17. Drew BJ, Adams MG, Pelter MM and Wung SF. ST segment monitoring with a derived 12-lead electrocardiogram is superior to routine cardiac care unit monitoring. Am J Crit Care . 1996;5:198-206.

Table 1. Subject Characteristics

\begin{tabular}{ll}
\hline Demographics & \\
Age, years (mean \pm SD) & $60.09 \pm 14.05$ \\
Female Sex, N (\%) & $14(42.42 \%)$ \\
BMI (mean \pm SD) & $29.97 \pm 7.00$ \\
Comorbidities & $21(63.64 \%)$ \\
Hypertension, N (\%) & $14(42.42 \%)$ \\
Hyperlipidemia, N (\%) & $16(48.48 \%)$ \\
Diabetes, N (\%) & $5(15.15 \%)$ \\
COPD/Asthma, N (\%) & $10(30.30 \%)$ \\
Chronic Kidney Disease, N (\%) & $4(12.12 \%)$ \\
Coronary Artery Disease, N (\%) & $7(21.21 \%)$ \\
Congestive Heart Failure, N (\%) & \\
Laboratory Values & $13.65 \pm 11.22$ \\
WBC Count (cells/uL) & $3.27 \pm 2.47$ \\
Creatinine (mg/dL) & $1992.24 \pm 1807.34$ \\
Ferritin (ng/mL) & $202.16 \pm 101.37$ \\
C-Reactive Protein (mg/L) & $84.30 \pm 28.29$ \\
ESR (mm/hr) & $8.26 \pm 6.67$ \\
D-dimer (ug/mL) & $715.30 \pm 869.22$ \\
Lactate Dehydrogenase (U/L) &
\end{tabular}


High Sensitivity Troponin-T (ng/L) $116.47 \pm 158.19$

Clinical Features

Mechanical Ventilation, N (\%) $33(100.00 \%)$

Renal Replacement Therapy, N (\%) $11(33.33 \%)$

Vasopressor Requirement, N (\%) $24(72.73 \%)$

Anti-arrhythmic use, N (\%) $3(9.09 \%)$

Anticoagulation use, N (\%) $8(24.24 \%)$

Hydroxychloroquine use, N (\%) $26(78.79 \%)$

Azithromycin use, N (\%) $27(81.82 \%)$

Table 2. Results of Measurements

\begin{tabular}{lll}
\hline & 12-lead ECG Manual Measurement & 12-lead ECG Computer Measurement \\
Rate (beats/min) & $85.49 \pm 22.35$ & $86.03 \pm 22.72$ \\
RR (msec) & $756.30 \pm 231.27$ & \\
QRS duration (msec) & $95.45 \pm 21.08$ & $93.76 \pm 21.12$ \\
PR duration (msec) & $166.45 \pm 26.02$ & $158.40 \pm 33.44$ \\
QT interval (msec) & $401.21 \pm 66.09$ & $398.60 \pm 64.80$ \\
Dispersion of QT (msec) & $37.88 \pm 16.73$ & \\
QTc (Bazett) (msec) & $467.49 \pm 50.91$ & \\
QTc (Fredericia) (msec) & $442.82 \pm 47.88$ & \\
QTc (Hodges) (msec) & $445.82 \pm 48.52$ & \\
QTc (Framingham) (msec) & $438.74 \pm 44.01$ & \\
\hline
\end{tabular}

Table 3. Mean Differences in Manual QT and QTc Measurements

\begin{tabular}{|c|c|c|}
\hline & Mean Difference between 12-lead ECG and 7-lead ECG & Mean Differenc \\
\hline 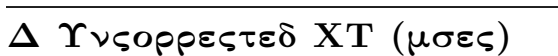 & $13.03 \pm 31.87^{*}$ & $19.09 \pm 33.67^{*}$ \\
\hline QTc (Bazett) (msec) & $13.45 \pm 32.05^{*}$ & $19.62 \pm 33.19^{*}$ \\
\hline$\Delta \mathrm{XT \varsigma}(\Phi \rho \varepsilon \delta \varepsilon \rho\llcorner\varsigma \iota \alpha)(\mu \sigma \varepsilon \varsigma)$ & $13.02 \pm 29.37^{*}$ & $19.22 \pm 30.85^{*}$ \\
\hline$\Delta \mathrm{XT \varsigma}(\mathrm{Ho \delta \gamma \varepsilon \varsigma )}(\mu \sigma \varepsilon \varsigma)$ & $13.08 \pm 26.97^{*}$ & $18.44 \pm 28.40^{*}$ \\
\hline$\Delta \mathrm{XT} \varsigma(\Phi \rho \alpha \mu \nu \gamma \eta \alpha \mu)(\mu \sigma \varepsilon \varsigma)$ & $10.73 \pm 26.51^{*}$ & $16.74 \pm 27.66^{*}$ \\
\hline
\end{tabular}

* = p-value $<0.05$ for a single sample t-test null hypothesis that the mean difference is equal to 0 .

$+=$ represents the $\mathrm{p}$-value for the two-sample paired t-test null hypothesis that the mean differences are equal to each other.

Table 4. Morphologic Features of ECGs

Rhythm, sinus rhythm, N (\%)

Rhythm, atrial fibrillation, $\mathrm{N}(\%)$

Rhythm, junctional rhythm, N (\%)

Axis, normal, N (\%)

Axis, left axis deviation, $\mathrm{N}(\%)$

Axis, right axis deviation, $\mathrm{N}(\%)$

Axis, northwest, N (\%)

Right bundle branch block, N (\%)

$\begin{array}{lll}\text { 12-lead ECG } & \text { 7-lead ECG } & \text { Telemetry Lead II } \\ 31(93.94 \%) & 31(93.94 \%) & 30(90.91 \%) \\ 2(6.06 \%) & 2(6.06 \%) & 2(6.06 \%) \\ 0(0 \%) & 0(0 \%) & 1(3.03 \%) \\ 29(87.88 \%) & 26(78.79 \%) & - \\ 3(9.09 \%) & 3(9.09 \%) & - \\ 1(3.03 \%) & 3(9.09 \%) & - \\ - & 1(3.03 \%) & - \\ 2(6.06 \%) & 2(6.06 \%) & -\end{array}$




\begin{tabular}{llll}
$\begin{array}{l}\text { Left bundle branch block, N (\%) } \\
\text { Intraventricular Conduction }\end{array}$ & $1(3.03 \%)$ & $1(3.03 \%)$ & - \\
Delay, N (\%) & $2(6.06 \%)$ & $1(3.03 \%)$ & $4(12.12 \%)$ \\
T Wave Inversion, N (\%) & $11(33.33 \%)$ & $14(42.42 \%)$ & $9(27.27 \%)$ \\
ST Elevation, N (\%) & $0(0.00 \%)$ & $5(15.15 \%)$ & $2(6.06 \%)$ \\
ST Depression, N (\%) & $1(3.03 \%)$ & $4(12.12 \%)$ & $1(3.03 \%)$ \\
Low Voltage QRS, N (\%) & $7(21.21 \%)$ & $2(6.06 \%)$ & $1(3.03 \%)$ \\
\hline
\end{tabular}

\section{Figure 1. Examples of 12-Lead and 7-Lead ECG Tracings}

Figure 1A represents a 12-lead ECG, with evidence of intraventricular conduction delay (IVCD) but no significant ST segment changes; Figure 1B represents the 7-lead ECG of the same patient, however without significant IVCD ad with 2-3mm ST segment elevation in the inferior leads and 1-2mm ST segment depression in leads I and aVL.

\section{Figure 1A}

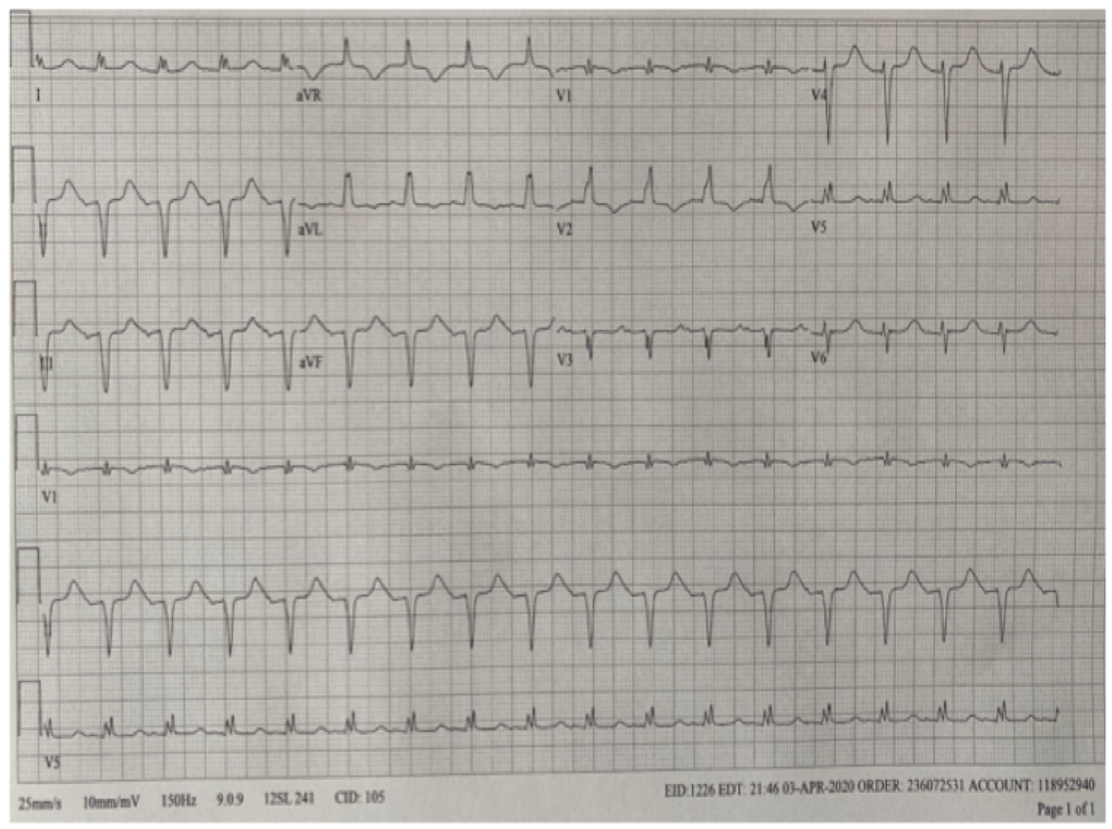

Figure 1B 


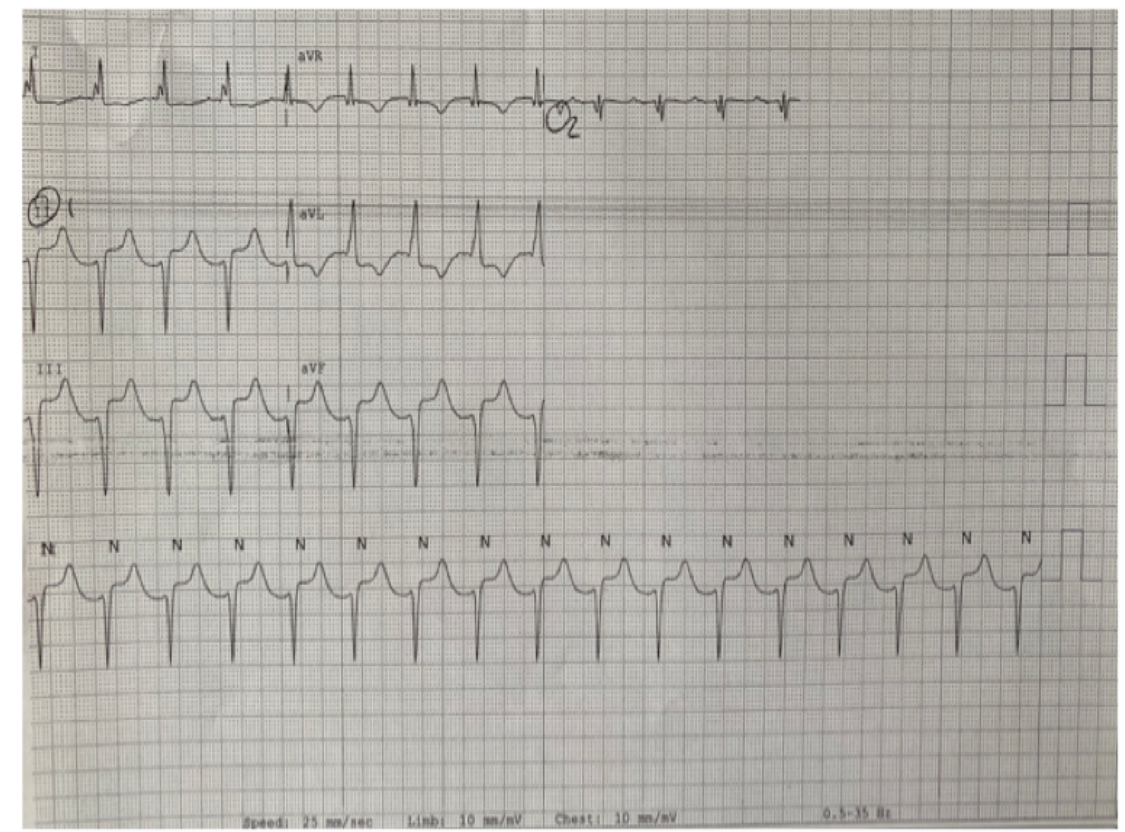

Figure 2: Scatter plot with $95 \%$ prediction ellipse showing linear correlation between 12-lead ECG QT measurement and telemetry QT measurement. Figure 2A Correlation between computer-measured QTc (Bazett) and manually-measured QTc (Bazett) on 12-lead ECG;

Figure 2B Correlation between manually-measured QTc on 12-lead ECG and 7-lead derived ECG; Figure 2C Correlation between manually-measured QTc on 12-lead ECG and lead II telemetry.

Figure 2A

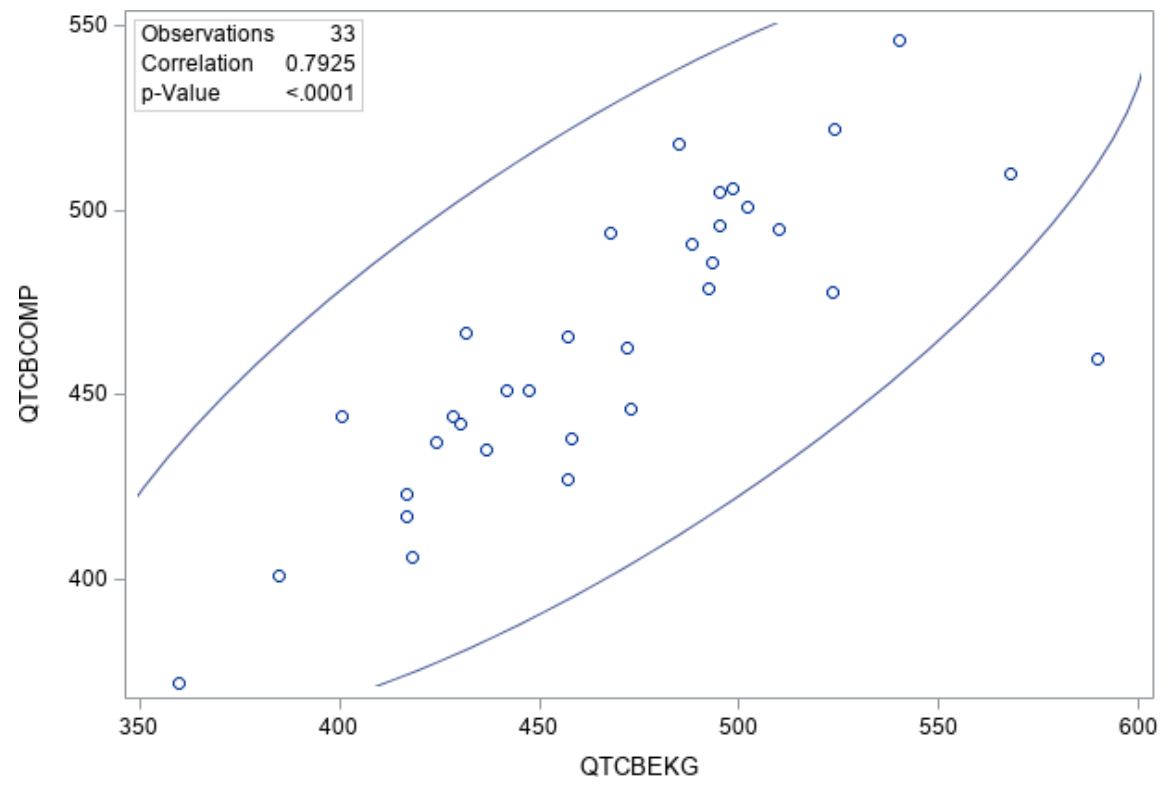

Figure 2B 


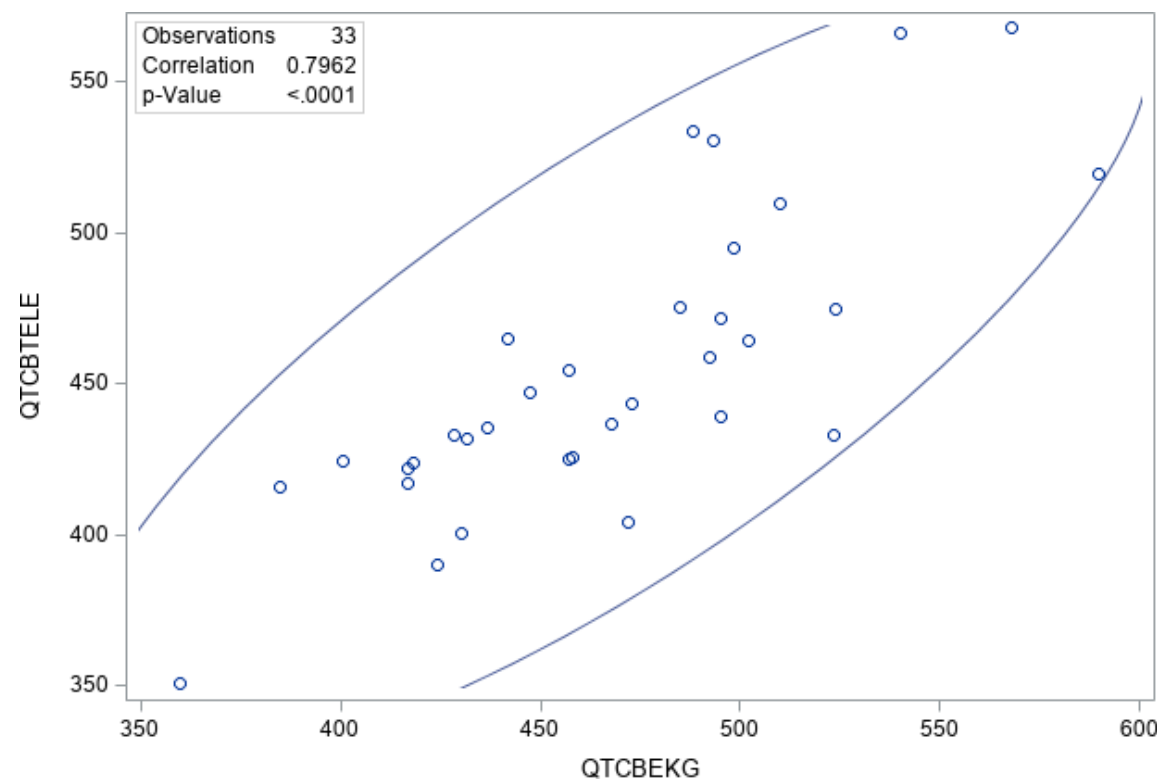

Figure 2C

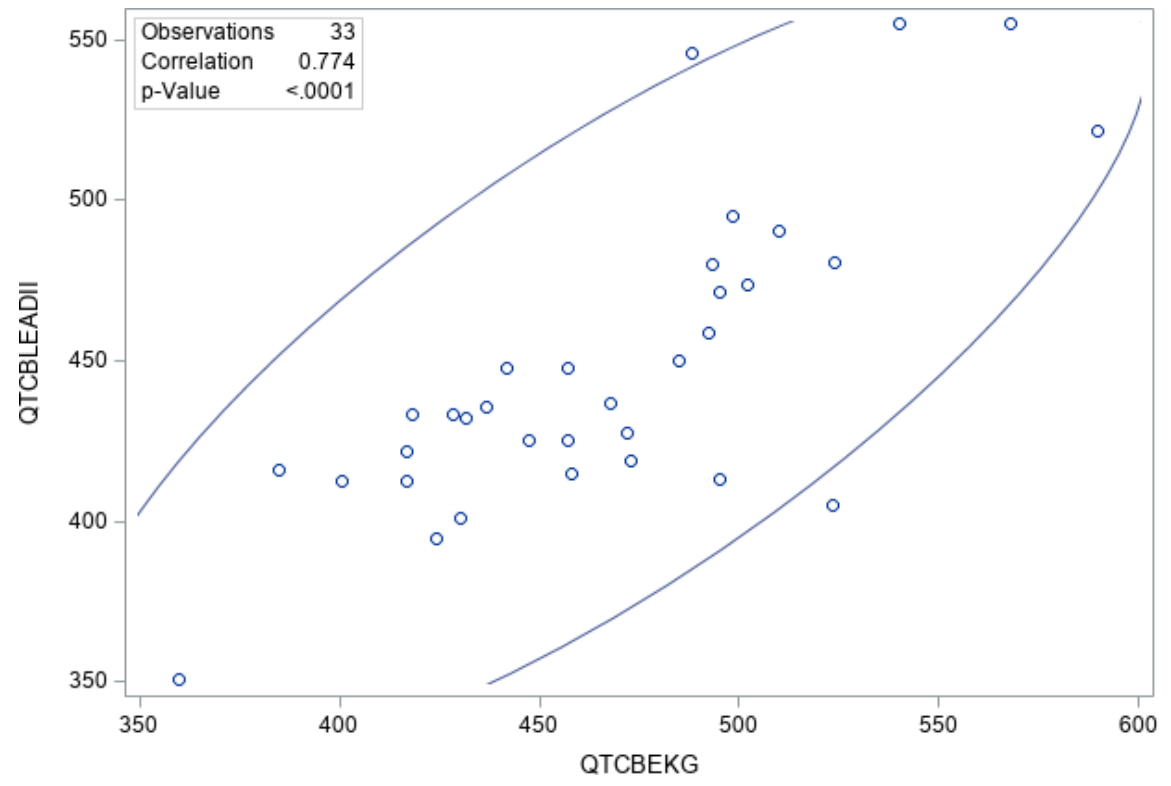

Figure 3. Bland-Altman plot demonstrating differences in between 12-lead QT measurement and telemetry QT measurement; Figure 3A. Bland-Altman Plot for differences in manually-measured corrected QT (Bazett) on 12-lead ECG vs. 7-lead derived ECG.Figure 3B. Bland-Altman plot for differences in manuallymeasured corrected QT (Bazett) on 12-lead ECG vs. Lead II Telemetry. In both plots, the solid blue line represents no bias. Solid red line represents mean bias between all measurements. Dashed red line represents \pm 2 standard deviations from the mean. Dashed purple line represents \pm 3 standard deviations from the mean.

Figure 3A. 


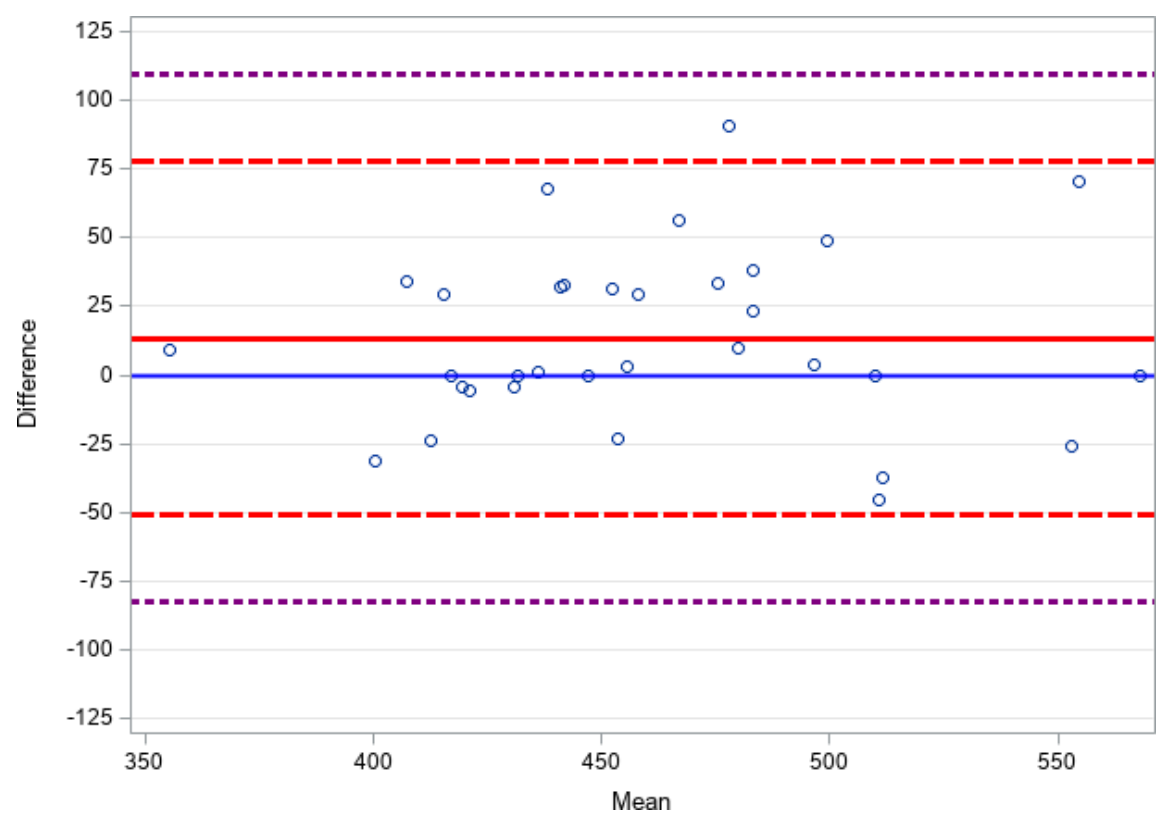

Figure 3B.

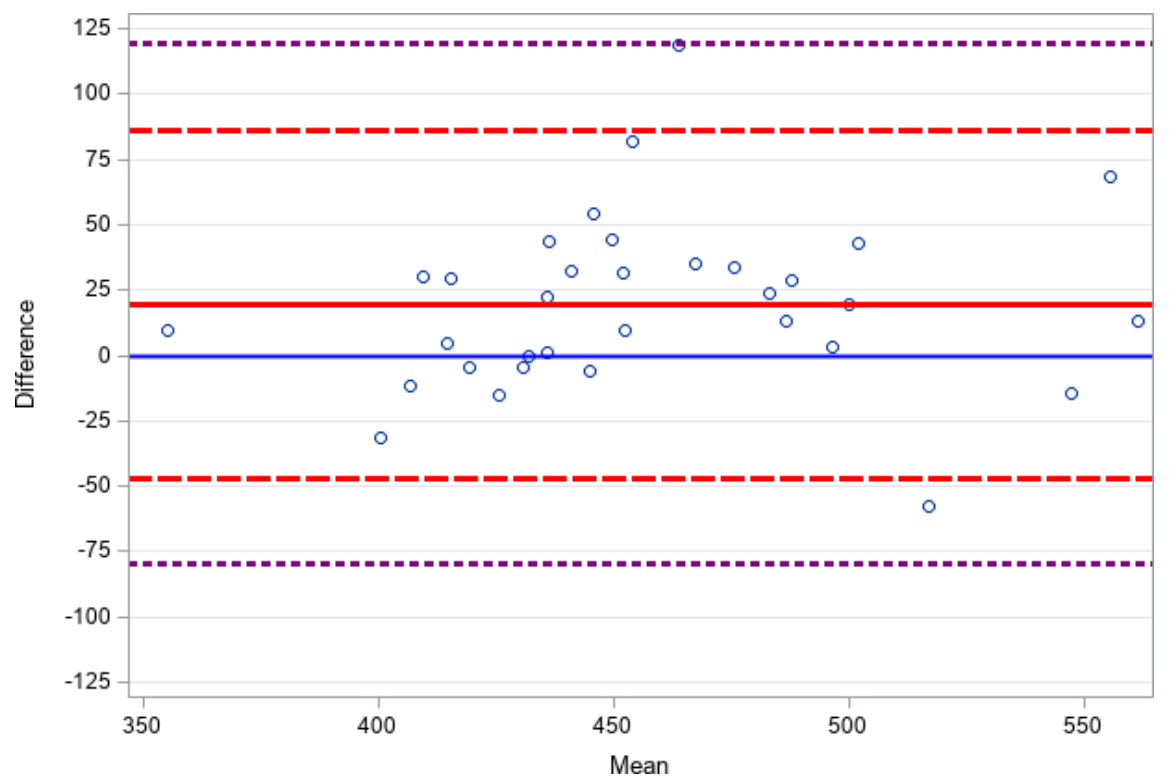

\section{SUPPLEMENTAL MATERIAL}

Supplemental Figure 1A. Scatter Plot with 95\% prediction ellipse showing linear correlation between computer-measured QT and manually-measured QT on 12-lead ECG 


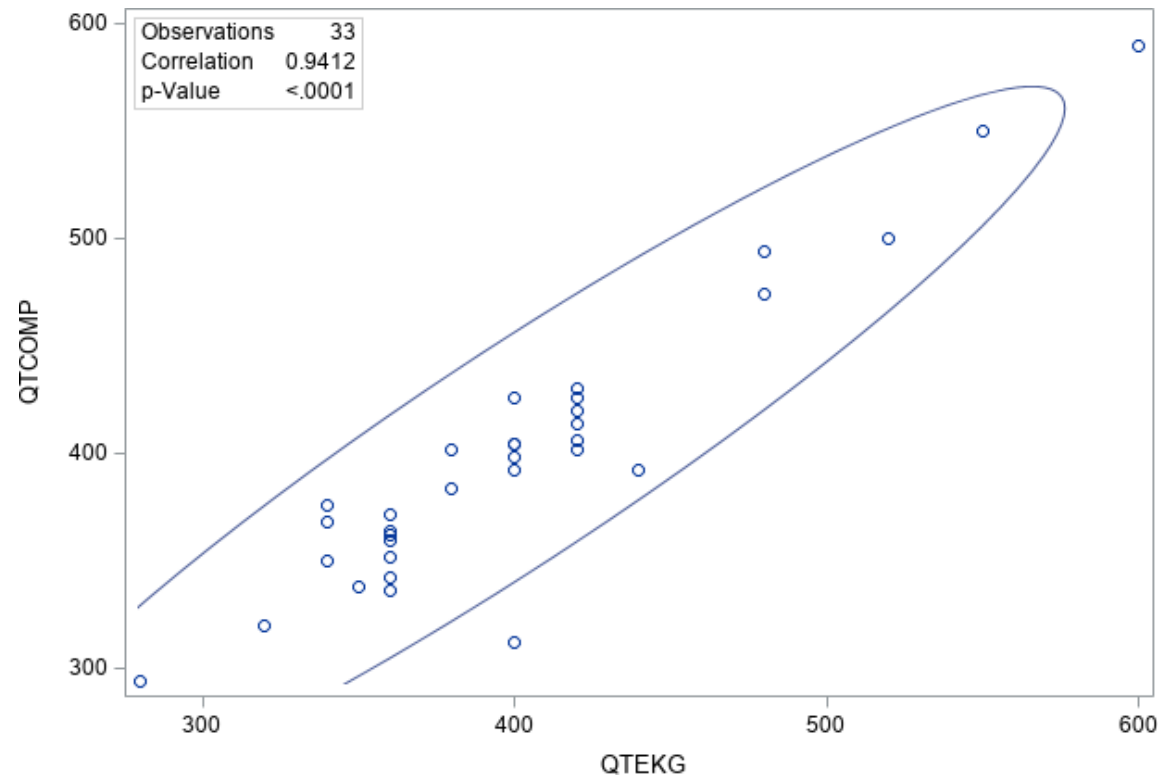

Supplemental Figure 1B. Scatter Plot with 95\% prediction ellipse showing linear correlation between manually-measured QT on 12-lead ECG and 7-lead derived ECG

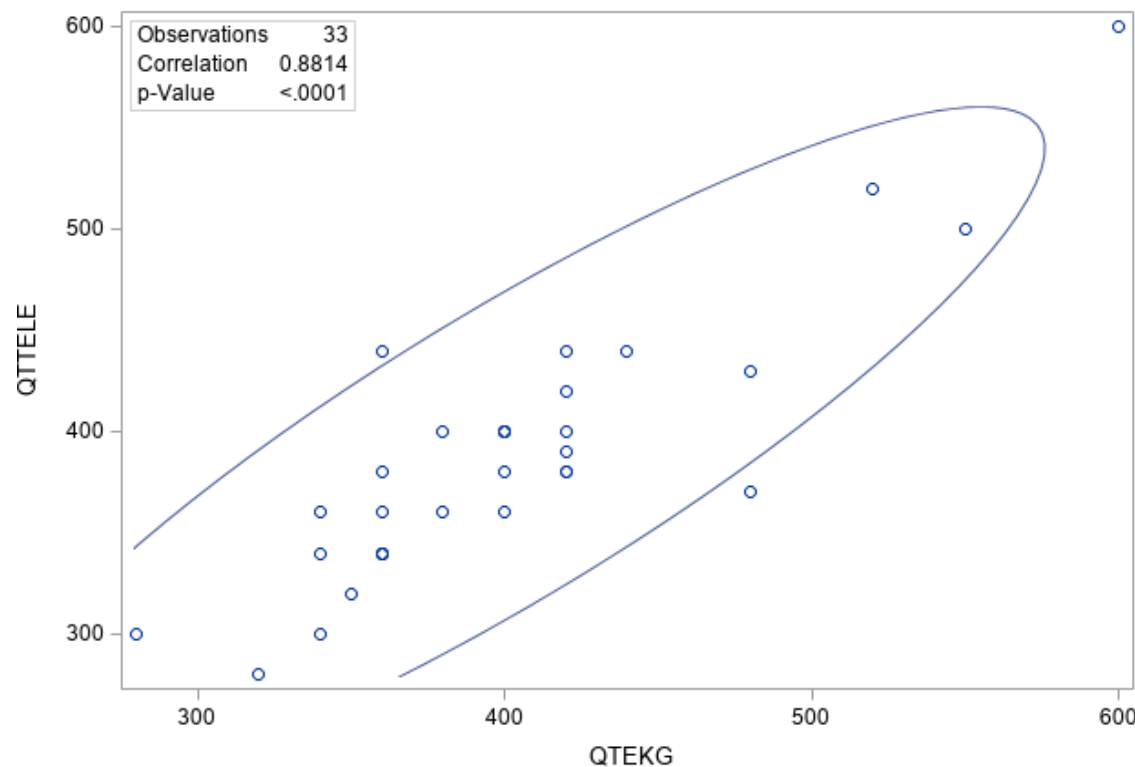

Supplemental Figure 1C. Scatter Plot with 95\% prediction ellipse showing linear correlation between manually-measured QT on 12-lead ECG and lead II telemetry 


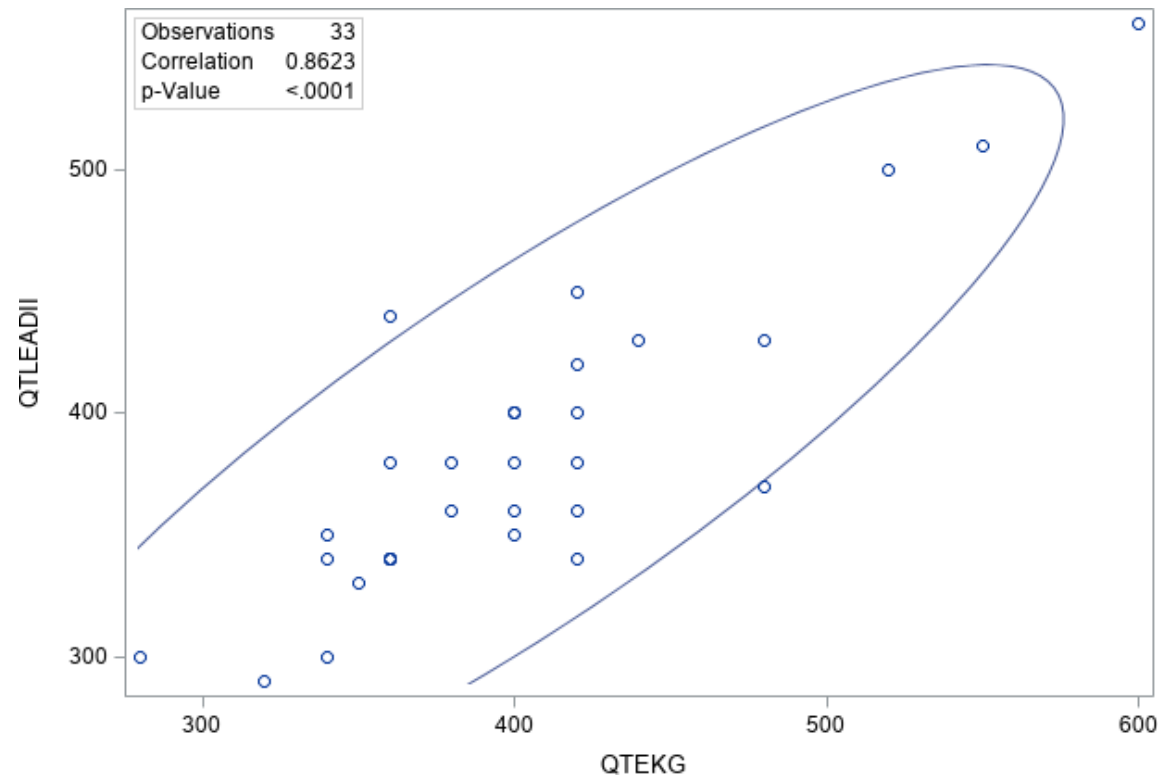

Supplemental Figure 1D. Scatter Plot with 95\% prediction ellipse showing linear correlation between manually-measured QTc (Hodges) on 12-lead ECG vs. 7-lead derived ECG.

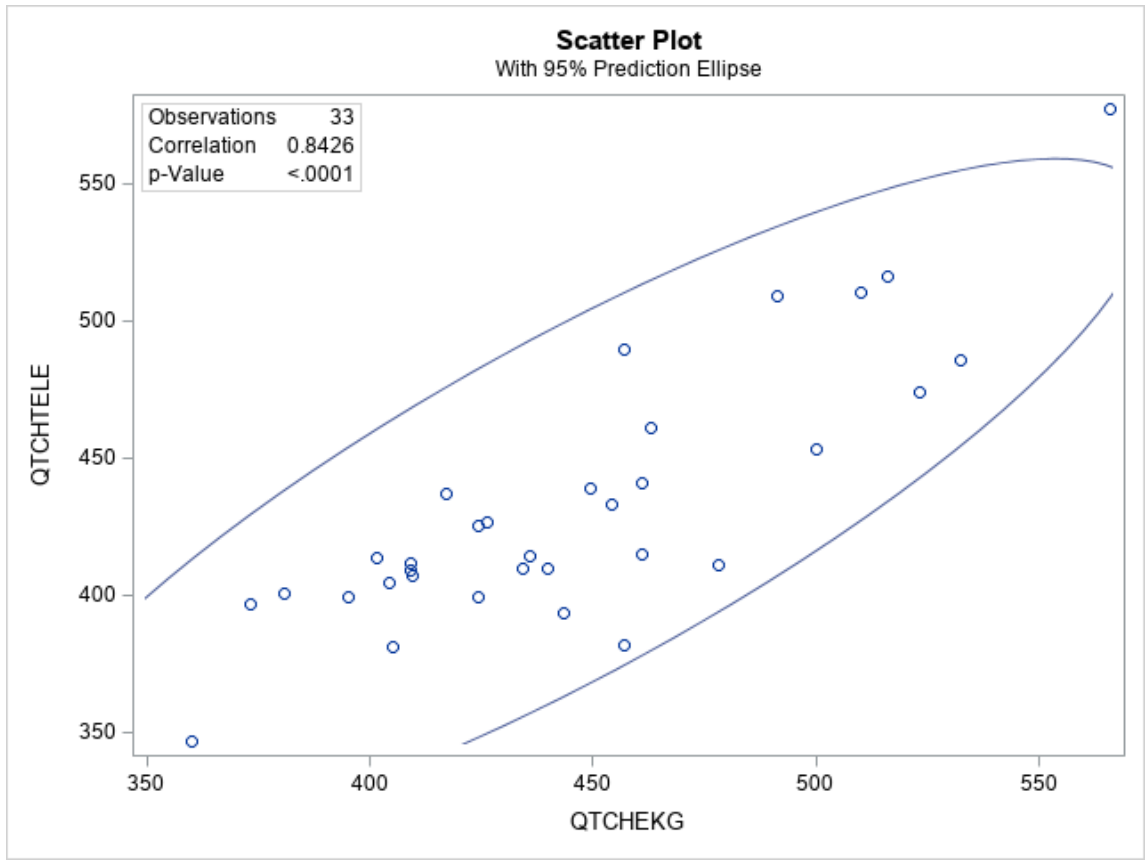

Supplemental Figure 1E. Scatter Plot with 95\% prediction ellipse showing linear correlation between manually-measured QTc (Hodges) on 12-lead ECG and lead II telemetry. 


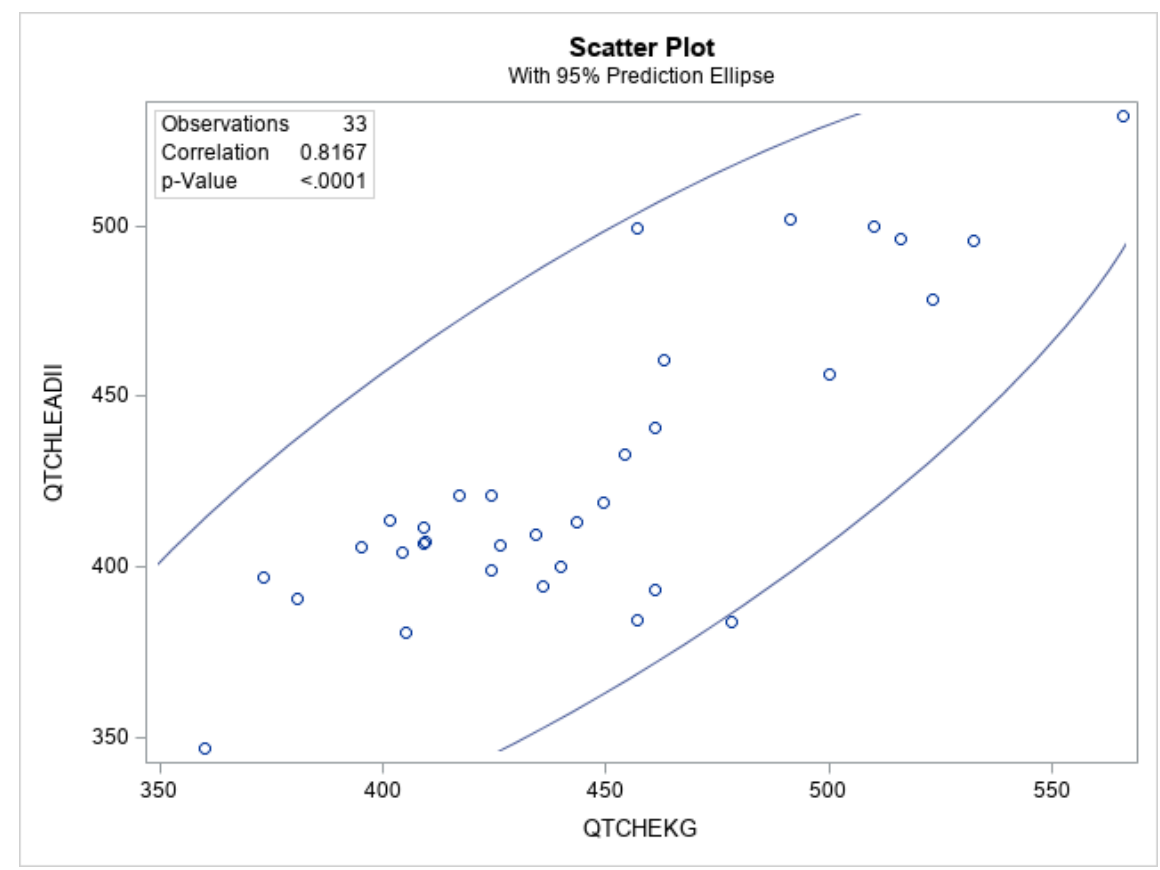

Supplemental Figure 1F. Scatter Plot with 95\% prediction ellipse showing linear correlation between manually-measured QTc (Fredericia) on 12-lead ECG vs. 7-lead derived ECG.

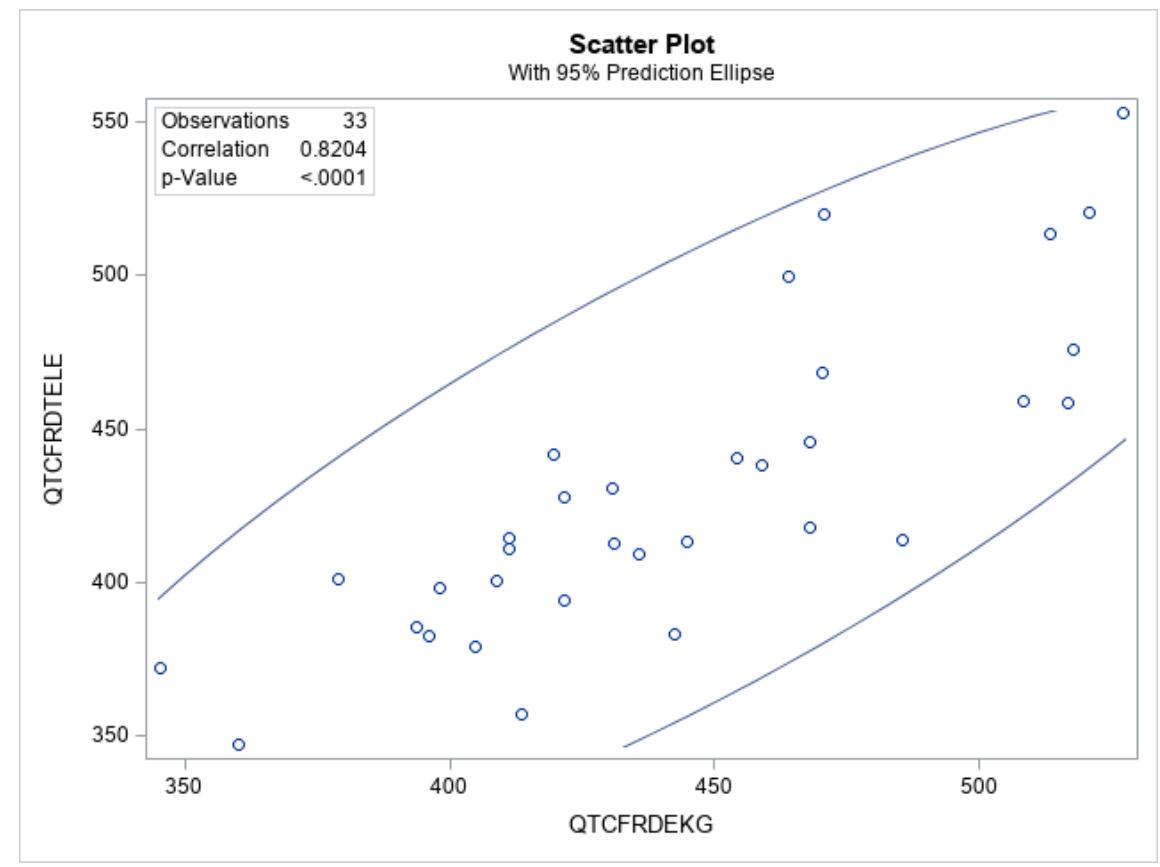

Supplemental Figure 1G. Scatter Plot with 95\% prediction ellipse showing linear correlation between manually-measured QTc (Fredericia) on 12-lead ECG vs. lead II telemetry. 


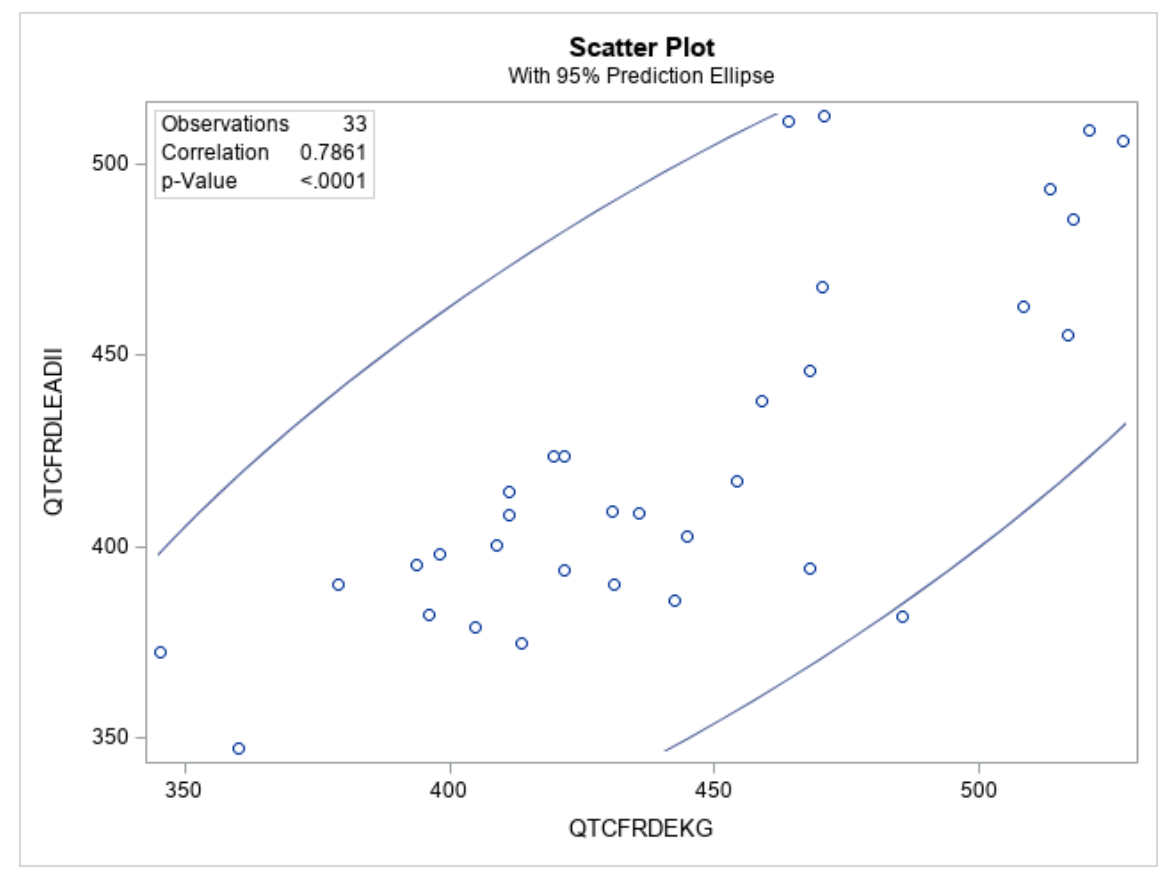

Supplemental Figure 2A. Bland-Altman plot for uncorrected QT on manually-measured vs. computer measured 12-lead ECG. Solid blue line represents no bias. Solid red line represents mean bias between all measurements. Dashed red line represents \pm 2 standard deviations from the mean. Dashed purple line represents \pm 3 standard deviations from the mean.

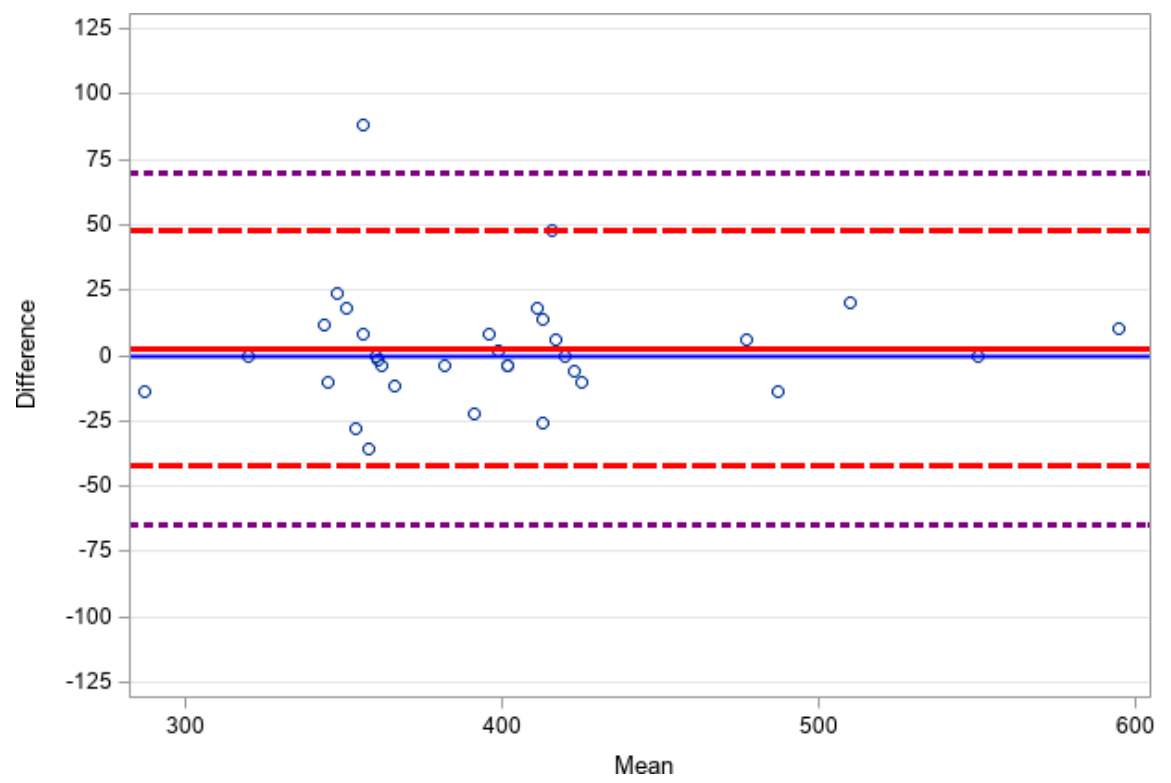

Supplemental Figure 2B. Bland-Altman Plot for uncorrected QT on manually-measured 12-lead ECG vs. 7-lead derived ECG. Solid blue line represents no bias. Solid red line represents mean bias between all measurements. Dashed red line represents \pm 2 standard deviations from the mean. Dashed purple line represents \pm 3 standard deviations from the mean. 


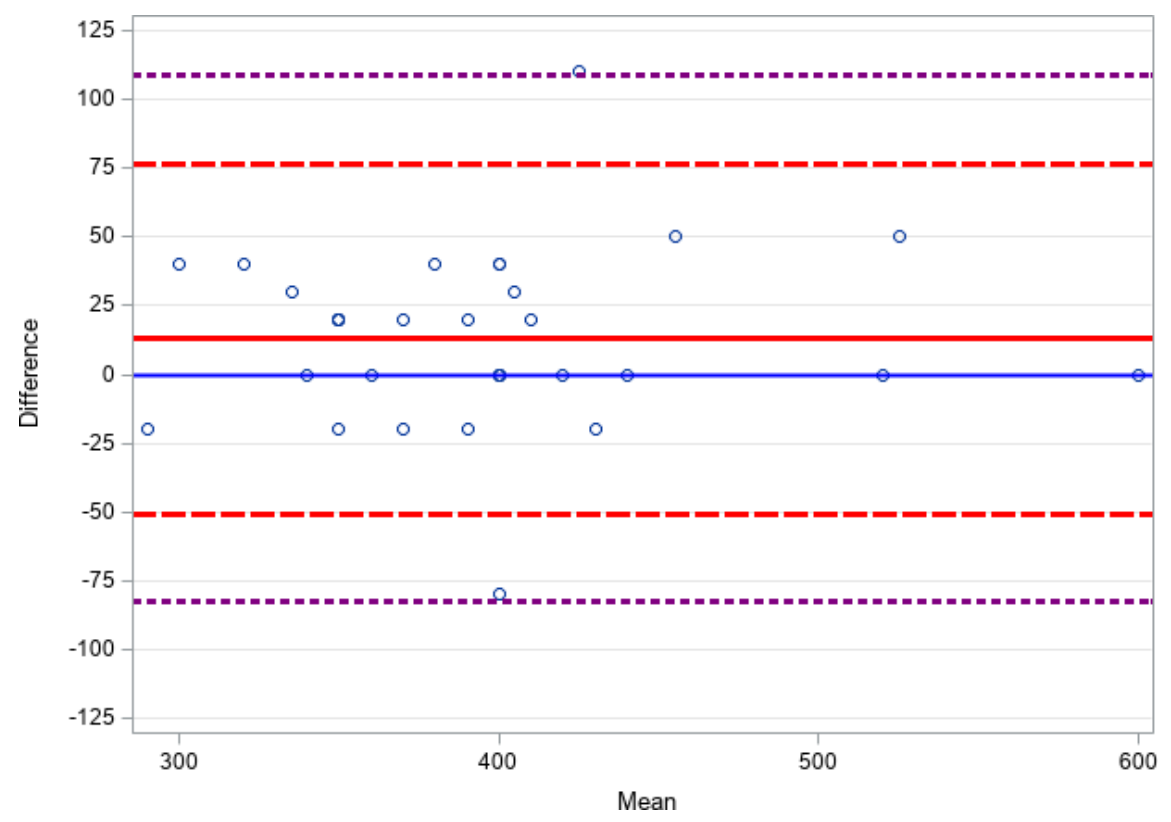

Supplemental Figure 2C. Bland Altman-Plot for uncorrected QT on manually-measured 12-lead ECG vs. lead II telemetry. Solid blue line represents no bias. Solid red line represents mean bias between all measurements. Dashed red line represents \pm 2 standard deviations from the mean. Dashed purple line represents \pm 3 standard deviations from the mean.

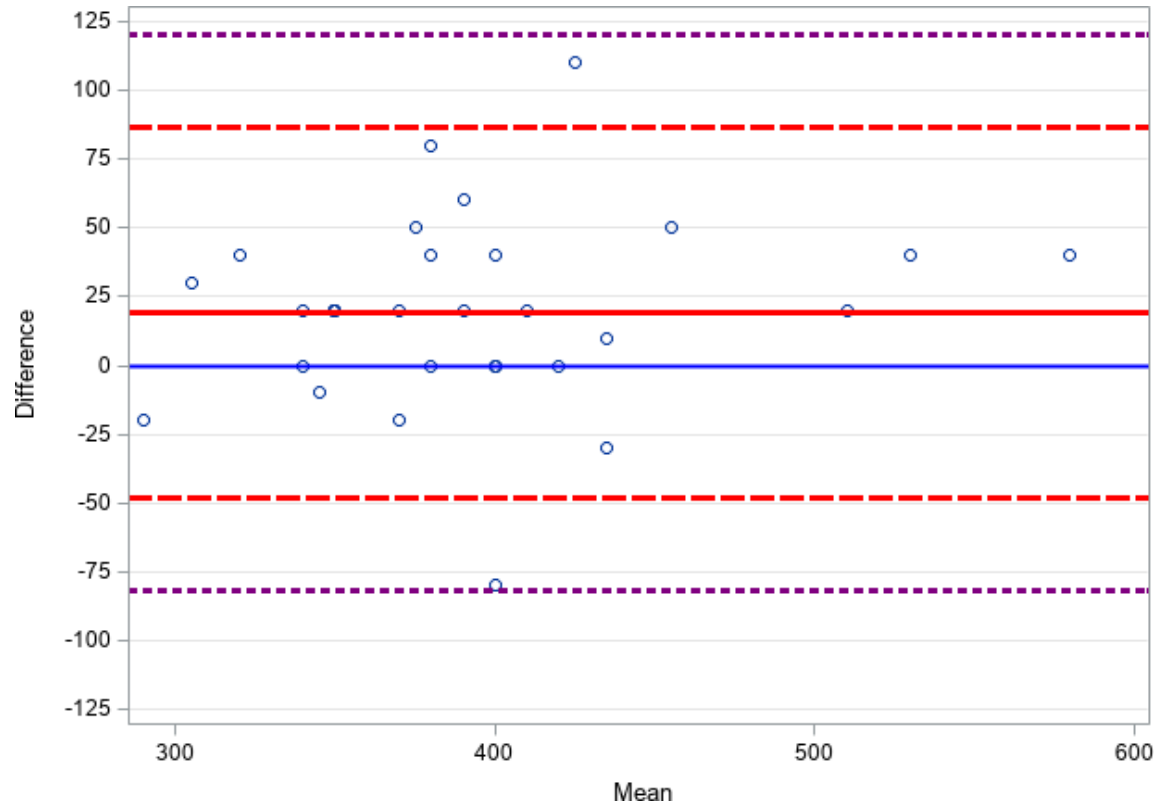

Supplemental Figure 2D. Bland-Altman Plot for QTc (Bazett) on manually-measured vs. computer measured 12-lead ECG. Solid blue line represents no bias. Solid red line represents mean bias between all measurements. Dashed red line represents \pm 2 standard deviations from the mean. Dashed purple line represents \pm 3 standard deviations from the mean. 


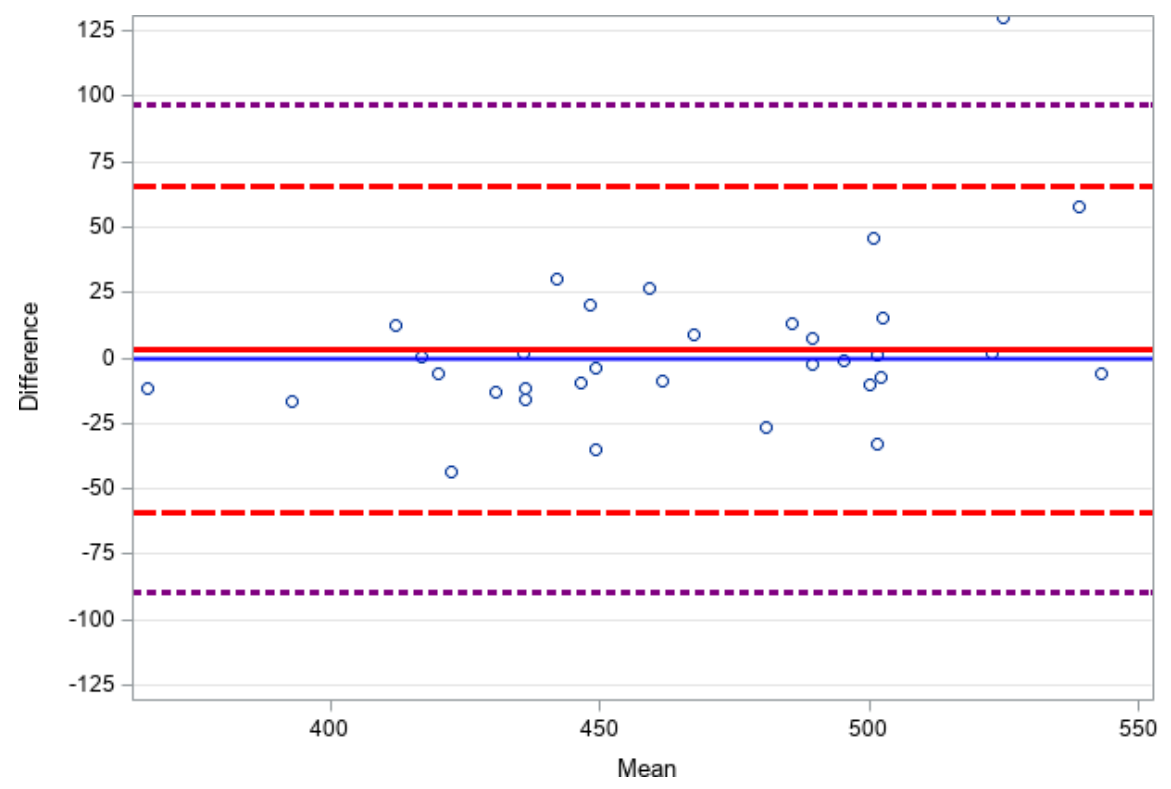

Supplemental Figure 2E. Bland-Altman Plot for QTc (Hodges) on manually-measured 12-lead ECG vs. 7-lead derived ECG. Solid blue line represents no bias. Solid red line represents mean bias between all measurements. Dashed red line represents \pm 2 standard deviations from the mean. Dashed purple line represents \pm 3 standard deviations from the mean.

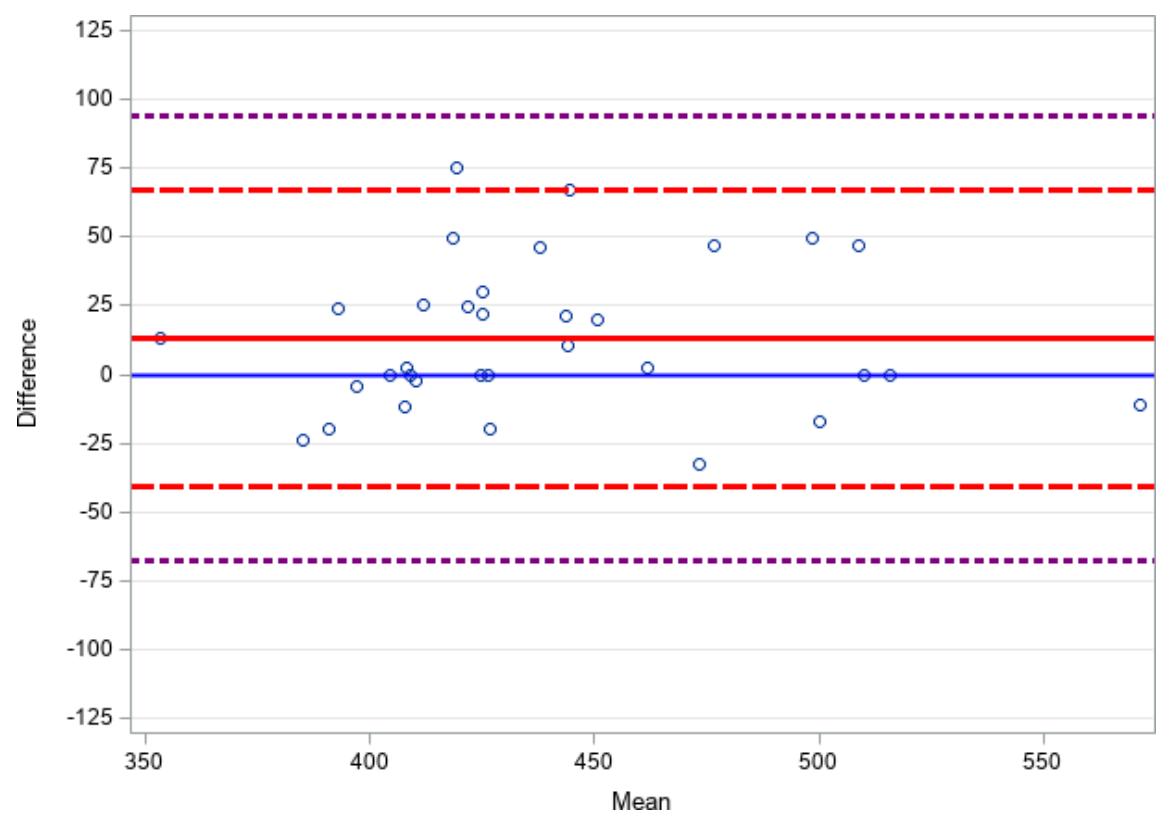

Supplemental Figure 2F. Bland-Altman plot for QTc (Hodges) on manually-measured 12-lead ECG vs. Lead II telemetry. Solid blue line represents no bias. Solid red line represents mean bias between all measurements. Dashed red line represents \pm 2 standard deviations from the mean. Dashed purple line represents \pm 3 standard deviations from the mean. 


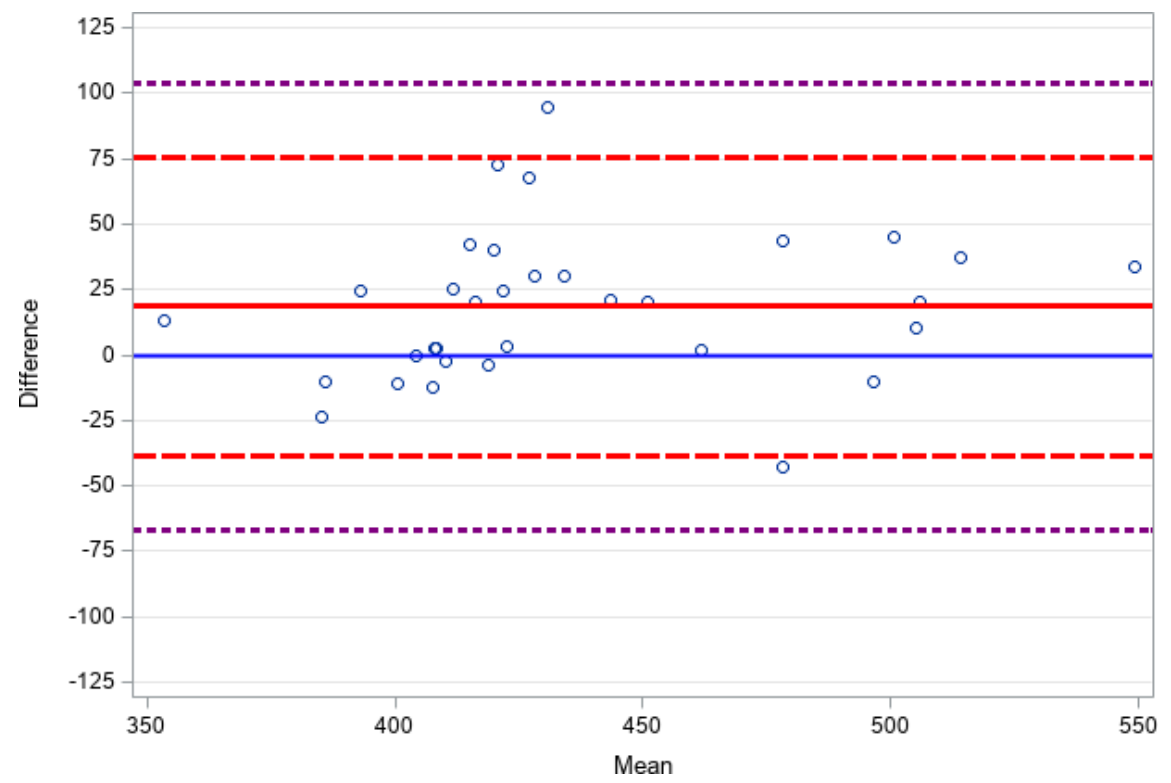

Supplemental Figure 2G. Bland-Altman plot for QTc (Fredericia) on manually-measured 12-lead ECG vs. 7-lead derived ECG. Solid blue line represents no bias. Solid red line represents mean bias between all measurements. Dashed red line represents \pm 2 standard deviations from the mean. Dashed purple line represents \pm 3 standard deviations from the mean.

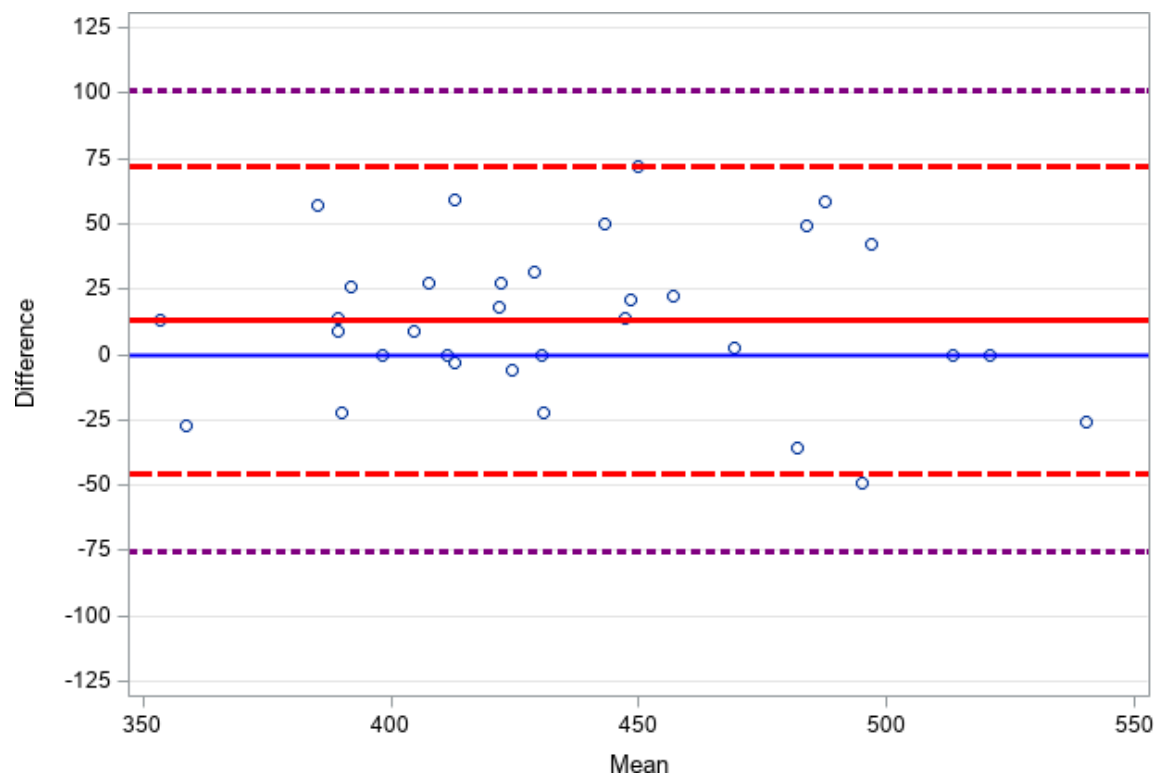

Supplemental Figure 2H. Bland-Altman plot for QTc (Fredericia) on manually-measured 12-lead ECG vs. lead II telemetry. Solid blue line represents no bias. Solid red line represents mean bias between all measurements. Dashed red line represents \pm 2 standard deviations from the mean. Dashed purple line represents \pm 3 standard deviations from the mean. 


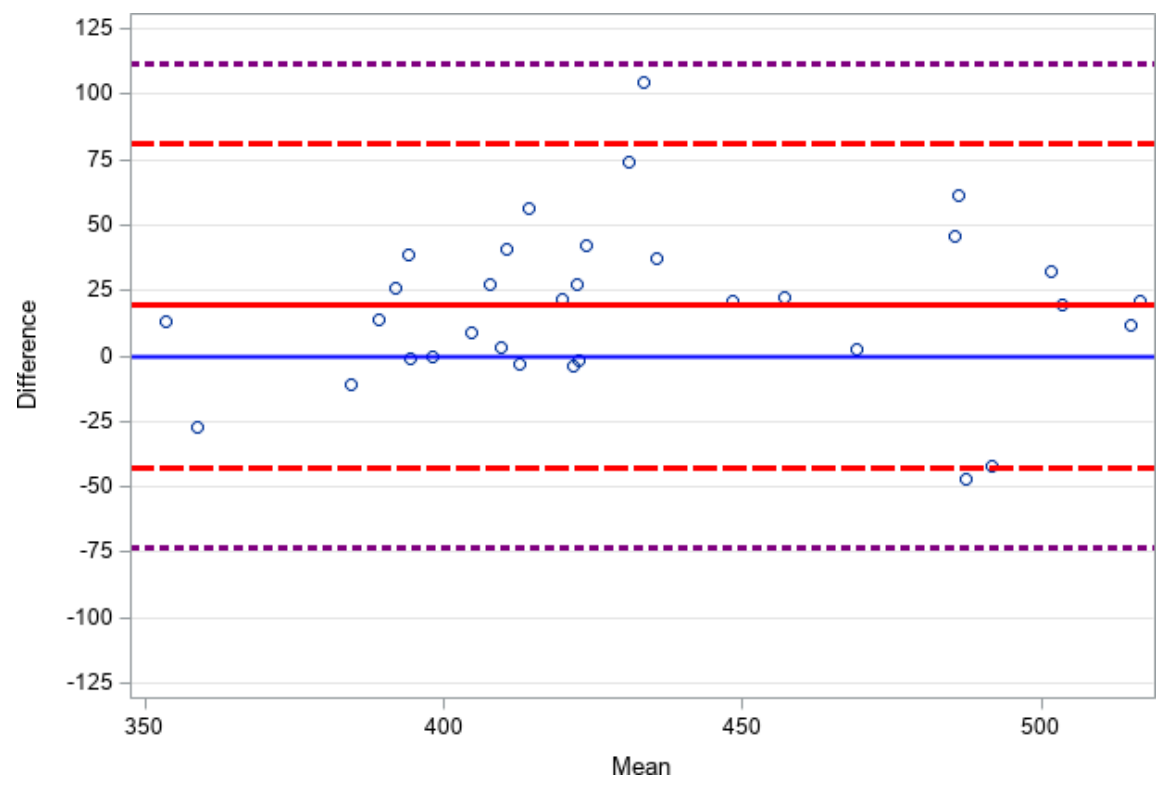

Supplemental Figure 2I. Bland-Altman plot for QTc (Framingham) on manually-measured 12-lead ECG vs. 7-lead derived ECG. Solid blue line represents no bias. Solid red line represents mean bias between all measurements. Dashed red line represents \pm 2 standard deviations from the mean. Dashed purple line represents \pm 3 standard deviations from the mean.

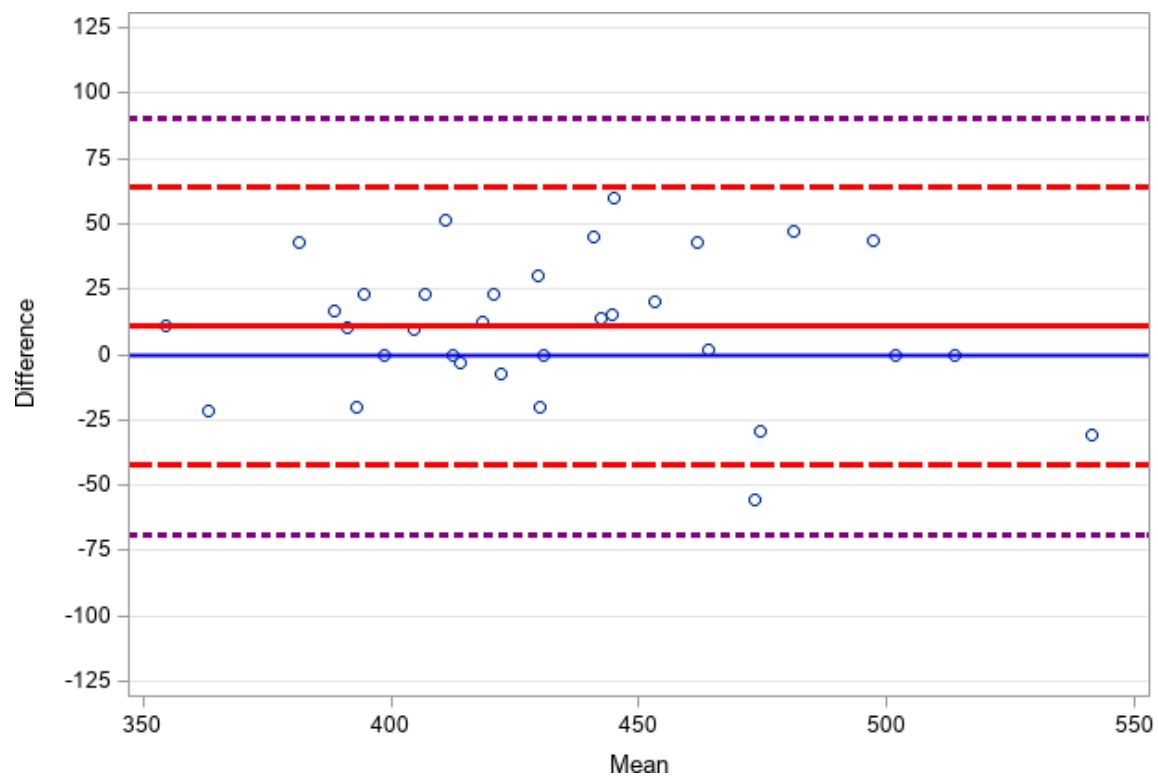

Supplemental Figure 2J. Bland-Altman Plot for manually measured QTc (Framingham) on 12-lead ECG vs. lead II telemetry. Solid blue line represents no bias. Solid red line represents mean bias between all measurements. Dashed red line represents \pm 2 standard deviations from the mean. Dashed purple line represents \pm 3 standard deviations from the mean. 


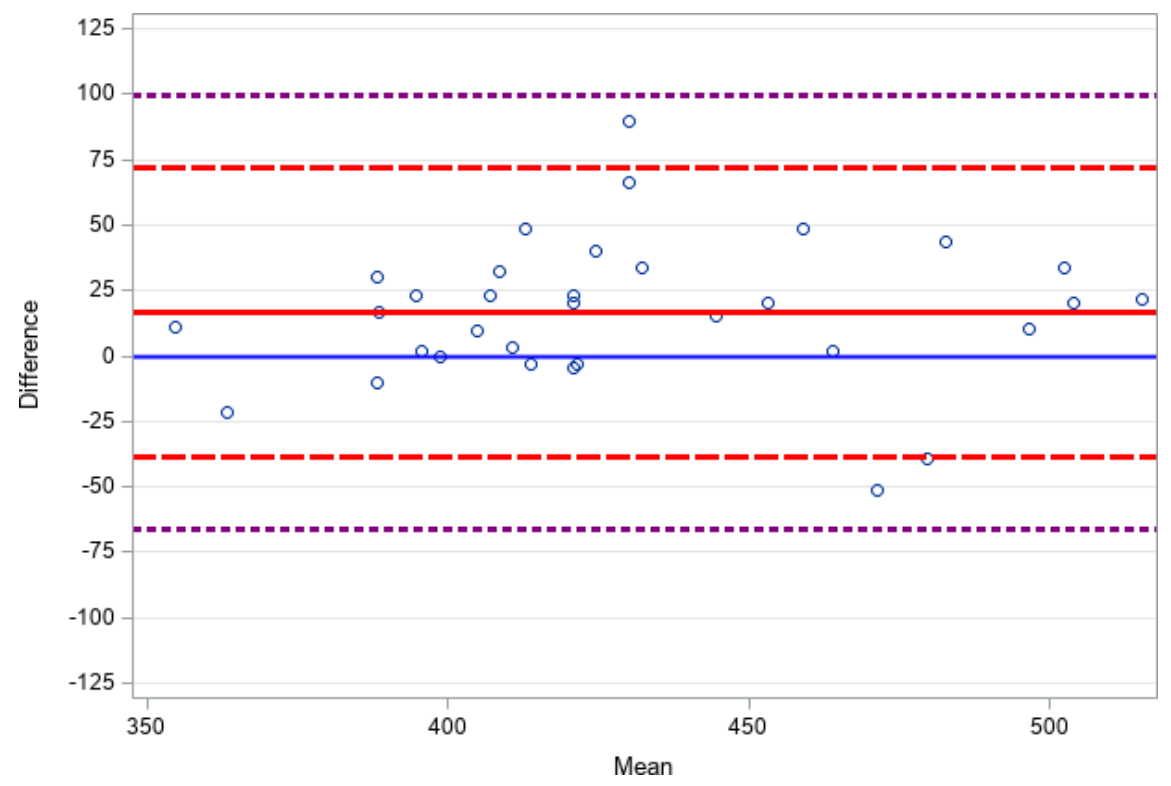

